\title{
Excess gestational weight gain: an exploration of midwives' views and practice
}

\author{
Jane C Willcox ${ }^{1 *}$, Karen J Campbell ${ }^{1}$, Paige van der Pligt ${ }^{1}$, Elizabeth Hoban ${ }^{2}$, Deborah Pidd ${ }^{3}$ \\ and Shelley Wilkinson ${ }^{4,5}$
}

\begin{abstract}
Background: Excess gestational weight gain (GWG) can affect the immediate and long term health outcomes of mother and infant. Understanding health providers' views, attitudes and practices around GWG is crucial to assist in the development of practical, time efficient and cost effective ways of supporting health providers to promote healthy GWGs. This study aimed to explore midwives' views, attitudes and approaches to the assessment, management and promotion of healthy GWG and to investigate their views on optimal interventions.

Methods: Midwives working in antenatal care were recruited from one rural and one urban Australian maternity hospital employing purposive sampling strategies to assess a range of practice areas. Face-to-face interviews were conducted with 15 experienced midwives using an interview guide and all interviews were digitally recorded, transcribed verbatim and analysed thematically.

Results: Midwives interviewed exhibited a range of views, attitudes and practices related to GWG. Three dominant themes emerged. Overall GWG was given low priority for midwives working in the antenatal care service in both hospitals. In addition, the midwives were deeply concerned for the physical and psychological health of pregnant women and worried about perceived negative impacts of discussion about weight and related interventions with women. Finally, the midwives saw themselves as central in providing lifestyle behaviour education to pregnant women and identified opportunities for support to promote healthy GWG.
\end{abstract}

Conclusions: The findings indicate that planning and implementation of healthy GWG interventions are likely to be challenging because the factors impacting on midwives' engagement in the GWG arena are varied and complex. This study provides insights for guideline and intervention development for the promotion of healthy GWG.

Keywords: Gestational weight gain, Pregnancy, Midwives, Weight, Qualitative research

\section{Background}

Over one third to a half of women enter pregnancy overweight or obese, with a Body Mass Index (BMI) $\geq 25 \mathrm{~kg} / \mathrm{m}^{2}$ [1-4]. This is compounded by $30-50 \%$ of women in developed countries, across all BMIs, exceeding recommended guidelines for gestational weight gain, (GWG) with the prevalence significantly increasing in the last two decades [5-8]. Many studies suggest that excess GWG, across all pre-pregnancy BMIs, is associated with short and long term negative health outcomes for maternal and child health $[9,10]$ and highlight the need for

\footnotetext{
* Correspondence: jwillcox@deakin.edu.au

'Centre for Physical Activity and Nutrition Research, School of Exercise and Nutrition Sciences, Deakin University, 221 Burwood Hwy, Burwood, Victoria 3125, Australia

Full list of author information is available at the end of the article
}

prevention. Excess GWG increases the likelihood of antenatal hypertensive disorders, gestational diabetes, atypical delivery outcomes and failure to breastfeed [9,11-15], and is associated with increased neonatal mortality, [16] and neonatal, infant and later life adiposity $[11,13]$. Further, increased and persistent postpartum overweight, higher weight in subsequent pregnancies and increased risk of overweight and obesity in later adult life are all associated with excess GWG, with each being a known risk for the development of cardiovascular disease and type II diabetes [5,9]. Currently there are no Australian GWG guidelines and the revised 2009 Institute of Medicine (IOM) guidelines [17] are most commonly adopted in developed countries in the absence of country specific guidelines $[17,18]$.

\section{Biomed Central}


Excess GWG is determined by a myriad of factors, including pre-pregnancy BMI, nutrition, physical activity, healthy pregnancy guideline awareness and psychosocial factors [17]. These predictors are potentially modifiable and opportunities exist to provide interventions to promote healthier GWG. Effective interventions may positively influence maternal and infant health outcomes, altering the future weight trajectory for two people and potentially impacting the intergenerational obesity cycle [19]. Evidence suggests that an antenatal intervention is opportune [20] and women are likely to be particularly receptive to advice during pregnancy [21]. However, resources in hospitals and in the community aiming to promote healthy GWG are limited [22]. A number of systematic reviews of interventions promoting healthy GWG have reported weak or inconclusive evidence regarding effectiveness [23-26], however recent meta-analyses report that interventions based on physical activity and diet counselling, combined with weight monitoring, appeared to be successful in reducing excess GWG $[27,28]$.

While the antenatal and delivery care for women will differ across countries and systems, midwives tend to be one of the key service providers, along with physicians and obstetricians [20,29]. Opportunities exist for midwives to support women to achieve positive lifestyle changes that may promote healthy GWGs $[18,20,30]$. Understanding midwives' views, attitudes and practices around GWG will provide insights to inform the development of practical, time efficient and cost effective ways of supporting health providers, including for midwives, to promote healthy GWGs with the potential to maximize best outcomes for pregnant women.

The research regarding midwives' views and practices concerning GWG is limited. A small number of studies have examined midwives and other health providers views, and approaches to antenatal weight measurement [18,31], services for obese women [32,33] or views of GWG management $[20,31,34,35]$. These studies report a broad range of views on weighing women, the importance of excess GWG and responses to excess GWG. Further, health providers were unsure of what to advise women regarding appropriate GWG and described barriers to engagement including insufficient training, concern about the sensitivity around weight, and the perception that counselling is ineffective. In many of the studies from the Australia [31], USA [20,34] and UK [32-34] midwives were a subset of these samples and only two UK studies have primarily sampled midwives [18,35]. In addition, the USA has GWG guidelines in place, whereas in Australia there are no GWG guidelines so it is important to assess midwives' views independently.

The aims of this study were to explore midwives': approaches to the assessment and management of healthy GWG; views on their role in identifying, managing and promoting healthy GWG and associated lifestyle factors and; views on optimal interventions to facilitate healthy GWG.

\section{Methods}

\section{Study design}

Qualitative descriptive research methodology [36,37] utilising face-to-face semi-structured interviews, using an interview guide were employed to obtain in-depth data from consenting midwives.

\section{Study participants}

The study recruited midwives who worked in, or managed, antenatal clinics in two maternity hospitals in Victoria, Australia, one rural and one urban. Purposive sampling [38] was used to ensure coverage of a broad range of practice areas. The sample of midwives sought included one Antenatal Clinic Director from each site and midwives across both hospitals. The sample size was informed by a similar study with general practitioners (GPs) that found data saturation occurred at 10-12 participants [39]. Midwives were invited to participate in interviews via written or face-to-face invitation after being informed of the study by their department managers.

\section{Data collection}

Face-to-face interviews with midwives were conducted by JW using a standardized interview guide (see Additional file 1). The content of the interview guides was informed by an analysis of the literature and a previous study with GPs [39]. The Antenatal Clinic Director's interviews aimed to provide an overview of the hospital context in relation to GWG that included policies, clinic guidelines and programs along with a personal perspective on GWG. Policies and guideline documents were examined during the interview with the Director in order to contextualize midwifery practice and provide an independent level of data for data triangulation [40] against the data collected from the midwives. Semistructured and structured questions to elicit midwives' views, attitudes and practices around GWG, as well as their thoughts on optimal interventions, were investigated during the interviews. Common themes explored included: focus of the first antenatal clinic visit; practice and advice regarding GWG and weighing; midwives perceived roles in lifestyle education, including GWG; and how midwives could be best supported to provide healthy lifestyle advice and support to pregnant women. In addition, socio-demographic characteristics of all participants were collected, which included the midwife's role in, and length of employment at the health facility. The interviews were digitally recorded, with the consent of the participants, and transcribed verbatim. Ethics approval was obtained from both hospital sites and Deakin University Human Research Ethics Committee. 


\section{Data analysis}

Data immersion, coding, category creation and thematic analysis were used to find repeated patterns of meaning across all data sets [41-44]. The researchers used an inductive approach using raw data to derive themes through interpretations made from the raw data [45]. Inter-rater reliability [46] was confirmed by two researchers carrying out the data analysis to reduce researcher bias during the thematic development phase. The final category system produced was agreed to by both researchers and accepted as being representative of the data.

\section{Results}

\section{Study participant characteristics}

Fifteen female midwives participated in the study. Three of the four possible midwives from the rural hospital and 11 of 25 midwives from the urban hospital consented and were interviewed. One additional midwife from the rural setting consented to be interviewed but withdrew due to illness. The Antenatal Clinic Director of the urban hospital (herself a midwife) was interviewed however, in the rural setting the midwives shared the administrative responsibility, and thus the administration related questions were shared among the participant midwives. Saturation of themes was evident after nine interviews, however the remaining interviews were carried out to ensure all practice areas were included and to confirm data saturation.

The midwives worked across a diverse range of antenatal practice areas including: hospital antenatal clinics $(\mathrm{n}=5)$; community outreach clinics $(\mathrm{n}=3)$; midwifery continuity clinics $(n=3)$; shared care (joint GP and antenatal clinic) $(n=1)$; perinatal clinic $(n=1)$; family birthing unit $(n=1)$ and Director of Antenatal Clinic $(n=1)$. The participants' experience working as a midwife averaged 21 years (range $3-37$ years). The interview length ranged from 30 minutes to 75 minutes.

\section{Emergent themes}

The thematic content analysis identified a number of overarching themes and subthemes. Three key themes emerged: 1. GWG being a low priority; 2. midwives concern for the physical and psychological welfare of women and; 3 . the central role for midwives in the education process with opportunities for additional support to promote healthy GWG. The Antenatal Clinic Director quotes have not been differentiated from the Midwife quotes due to the possibility of interviewee identification and thus breach of anonymity.

\section{Theme 1: Gestational weight gain is a low priority for midwives}

With the many competing interests in antenatal clinics, GWG was perceived by many midwives to be of low priority. A range of factors contributed to this perception, ranging from absence of policies through midwife beliefs regarding GWG, and their support to engage effectively on this topic. Contributing factors included: practices, policies and views limiting the weighing of women and provision of GWG guidelines; perceptions regarding pregnant women's low levels of interest in weight; limited education of midwives regarding GWG; time limitations for education of pregnant women; and perceptions of limited allied health services, such as Dietetic and Physiotherapy resources.

\section{a. Low incidence of weight monitoring}

In both hospitals midwives often weighed women at the first antenatal visit, and sometimes BMI was calculated, primarily as a risk stratification strategy. Generally, a woman's weight was not re- measured during her pregnancy unless the woman was defined as "high risk" (BMI $>35 \mathrm{~kg} / \mathrm{m}^{2}$ or presenting with a co-morbidity) at the outset. The urban hospital had a formal weighing policy [47] and GWG guidelines available to staff on the internal intranet. This hospital's policy and practice at the hospital discouraged weighing women after the first antenatal visit and this was reflected in some midwives' views. Further, the policy encouraged the provision of the IOM GWG guidelines based on BMI [17]. Despite the presence of weighing and GWG guidelines policy in the urban hospital and the absence in the rural hospital, there did not appear to be a significant difference in views and practices between both midwife groups. Both groups exhibited diverse views and practices.

\section{" (I don't think weighing is) relevant; we're just going \\ by clinical indications." (Urban midwife 2)}

When midwives were asked about routine weighing practices, two-thirds said they did not consider that routine weighing of pregnant woman was important. The midwives stated there was "no evidence" to support routine weighing and that measurements did not provide useful clinical information. In addition, midwives reported feeling that routine weighing may cause women psychological distress. The acknowledgement of change in pregnant women's weight was seen to come primarily from the women or midwives' observation.

'... the research supports that they don't really need to be weighed at every appointment. It doesn't really gain much information out of it." (Urban midwife 9)

"Too much stigma associated with it. . It's embarrassing for the patient; they see it as a kind of 
test, how good they've been or how bad they've been." (Urban midwife 7)

". . here we can provide continuity of care, so I can actually see the same women for all of her appointments apart from one or two because she's seeing her doctor. So I can actually gauge them (visually), how much they've been putting on." (Urban midwife 9)

However, the remaining third of midwives supported weighing during pregnancy and felt that weighing women at each antenatal visit allowed them to track GWG, particularly in high risk women such as those of high and low BMIs or those at risk of weight loss. Recording routine weights on women's care plan was seen as a practice that would normalise weighing and help trigger conversation with women regarding weight and lifestyle behaviours.

"We were told that by weighing women, it doesn't tell us about good foetal outcomes, so we stopped... But we forgot about the process for women, and what are the outcomes for women if we do weigh them and know what weight they are at the end of the pregnancy." (Urban midwife 6)

"(weighing)... instigates a conversation sometimes at each visit, whereas here women don't get weighed as a general rule. ... so you don't have that conversation, or you don't have that prompting." (Urban midwife 5)

The challenges associated with the identification of abnormal weight changes were raised by a few urban midwives. They acknowledged that weight changes cannot be identified when women are not weighed routinely.

". . they're not identified. We would have no idea what people put on in pregnancy." (Urban midwife 5)

b. Diverse views regarding provision of pregnancy GWG guidelines

Midwives expressed mixed feelings regarding whether GWG guidelines should be provided to women. Twothirds of the midwives indicated they did not consider it necessary to provide pregnant women with GWG guidelines unless the woman asked for them. Midwives' reluctance to discuss weight reflected a perceived lack of evidence regarding GWG, weight not being a priority for the midwives and concerns that women may become fixated on their weight during pregnancy. Consistent with the views regarding routine weighing, high risk women with high BMI and concurrent diseases such as diabetes were seen to be the exception.
"...I think the trend is not worry so much how much weight gain you have right through unless there's other medical issues involved such as hypertension and smoking and all that side issues." (Rural midwife 2)

"I guess it's that thing where you know the woman is going to put on weight and they do eat more so generally I guess I wouldn't feel that I would need to." (Rural midwife 3)

"(Providing guidelines).. should be always research based, but I don't think it is." (Urban midwife 6)

The third of midwives who provided GWG guidelines to women cited foetal and maternal outcomes as their main reason for doing so, along with the habit of providing weight guidelines to women.

"I feel that they all should be given so that they have a rough idea of what is normal and not normal, so they're having a proper diet and exercise." (Urban midwife 2)

"So I think we ...... need some guidelines" (Rural midwife 2)

The GWG guidelines provided to women during antenatal care by all midwives, either voluntarily or if asked, varied greatly. A few urban midwives provided women with individual guidelines related to pre-pregnancy BMI, such as the IOM guidelines [17], but the majority provided highly varied ranges for example $10-20 \mathrm{~kg}$ or 10 $14 \mathrm{~kg}$. Not providing GWG information to women was in contrast to the policy supporting the provision of GWG guidelines at the urban hospital [47].

Half the midwives said that women sought weight gain advice during antenatal care and half noting that weight was rarely raised in consultations. Some midwives felt that healthy weight women were more likely to ask about GWG guidelines. A few midwives shared their personal strategies on discussing GWG and normalising the healthy GWG. The most common strategies involved focusing on the benefits of healthy GWG for the foetus and differentiating the pregnancy weight gain from weight gained through a positive energy balance.

"I tell them that gaining weight in pregnancy is completely different to gaining weight when you eat too much cake." (Urban midwife 7)

c. Excess GWG not seen to be common or problematic by many

Most midwives considered excess GWG to be uncommon with the exception of women deemed at "high risk". In 
addition, many communicated that they did not see excessive GWG as a significant health issue for women. However, it was also highlighted by some that GWG was impossible to detect since weighing pregnant women was uncommon.

"(Excess GWG) is unusual from my experience..." (Urban midwife 1)

“. . your baby's an appropriate size then no-one's going to be too concerned if there's a 20 kilo weight gain." (Urban midwife 5)

". . they're not identified. We would have no idea what people put on in pregnancy." (Urban midwife 5)

In contrast, the midwives who considered excessive GWG problematic were concerned about maternal and foetal outcomes. There was a sense that the emphasis on GWG had been inappropriately played down over recent years. In addition, concern was expressed that excess GWG compounded associated problems for those already overweight or obese.

"But I think we sort of ignore the fact that a lot of the girls have started heavier. We are a fatter population so we still have the problem of really big women being pregnant, and getting to the end of the pregnancy they have other problems as well they get too big." (Rural midwife 2)

When midwives were prompted to identify important implications of excess GWG, the most common responses related to gestational diabetes, preeclampsia, inability to palpate the foetus and complicated deliveries. Two midwives mentioned foetal health implications, including macrosomia.

\section{d. Limited resources to address GWG and lifestyle behaviours}

The midwives identified a lack of time and resources, such as dietetic services, as key limitations enabling them to address healthy GWG and lifestyle issues with the women. Midwives are required to address a large number of issues during antenatal consultations including assessment of medical, family, pregnancy and psychological history as well as provision of pregnancy information, antenatal tests, procedures and bookings. Midwives considered they had limited time available for discussions about GWG and healthy lifestyle. The late timing of the first antenatal visits (often occurring after the first trimester) was sometimes seen to preclude education when it would have been most appropriate. In addition, a reluctance to bombard women with excess information influenced midwives' decisions about what topics to discuss during visits.
“.. when you're on a time efficiency. ...you can't really think of every topic, because every topic in pregnancy has become the most important, because there's always a smoking process going on. There's the alcohol intervention process, so everything becomes the most important thing in pregnancy." (Urban midwife 5)

"..they are blown away by how much we give them in the early visits." (Urban midwife 1)

As noted, the limited resources for dietetic and physiotherapy services were seen to constrain interventions for healthy GWG and lifestyle issues, reducing the ability for antenatal services to intervene even if a need was identified.

“.our Dietetics have an appointment system ..(and).. those appointments are hard to get because they take a long time and by the time you get there you could be half way through the pregnancy. " (Urban midwife 10)

One midwife felt that the limited dietetic and physiotherapy resources available to them in the public health system has resulted in a redefinition of "at risk" or "healthy" pregnancy weight because only those women with BMIs $>35 / \mathrm{kg} / \mathrm{m}^{2}$ were chosen for interventions and education. Therefore women with BMIs $25 \mathrm{~kg} / \mathrm{m}^{2}$ to $35 \mathrm{~kg} / \mathrm{m}^{2}$ were redefined as "normal".

“..there's a lot of issues for women around ...being fat and weight gain and pregnancy which we normalise" (Rural midwife 1)

\section{Theme 2}

\section{Concern for physical and psychological health of pregnant women}

Midwives articulated a concern for the physical and psychological health of pregnant women in general. However, their greatest concern was for possible psychological ramifications of weight related discussions and interventions.

a. Concern for the psychological impacts of weight discussions and women's inappropriate views on weight gain

It was a common view among the midwives that many women were inappropriately concerned about putting on too much weight during pregnancy. This concern was mirrored in the antenatal weighing policy of one hospital [47]. At this urban hospital the midwives felt that women were controlling their GWG through inappropriate strategies, such as restricted eating, but did not cite evidence to support the supposition. Hence, with a desire to "do no harm" some midwives were concerned about perceived psychological ramifications if weight and GWG were discussed and monitored at 
routine antenatal visits. Further, there was concern that women would become anxious about their weight, or actively lose weight which would have adverse effects on the mother and foetus. This was expressed as the prime reason for not discussing GWG.

"I think it stresses a lot of pregnant women out. I find a lot of women are fixated on weight and how much they should be gaining." (Urban midwife 3)

"Women were getting very anxious and they were getting obsessed about (weight gain) and I think that added extra anxiety, they're already anxious with their pregnancy." (Urban midwife 2)

Other midwives recognised the co-morbidities associated with excessive GWG, such as poor delivery and foetal outcomes, caused by not informing and/or supporting women to achieve these goals and the need for good health outcomes.

"You know, we can be nice about it all, but at the end of the day, we want good foetal, good maternal outcomes." (Urban midwife 6)

b. Concern for the physical health of women

The majority of midwives expressed deep concern about the physical health of their patients. In particular, a few expressed concerns about the increasing incidence in overweight and obesity in the community and their desire for an intervention to reduce women's weight prepregnancy.

"I consider it (pre-pregnancy overweight and obesity) a really big (issue), probably across my midwifery time one of the biggest issues that's out there at the moment." (Urban midwife 7)

\section{Theme 3}

Midwives are central to healthy lifestyle education process and opportunities exist for support to promote healthy GWG

All midwives viewed themselves as part of a team of antenatal colleagues who were responsible for the promotion of healthy lifestyle behaviours, including healthy GWG. When asked about how midwives could be best supported to deliver healthy weight and lifestyle behaviour education, a number of models were suggested.

\section{a. Key providers of lifestyle behaviour education}

Despite some midwives expressing concerns about healthy GWG and their role in its promotion, the midwives unanimously saw themselves as having responsibility for education and interventions around GWG and lifestyle issues. This was seen to be a responsibility shared with obstetricians, general practitioners and other health providers that pregnant women consulted. The need for consistent messages and education along with multidisciplinary care was also mentioned.

"So it's all our jobs and the idea would be to work together and with our most difficult clients using support such as Dietetics and whatever it is the woman needs." (Urban midwife 7)

Most midwives discussed some lifestyle behaviours during pregnancy, however, they considered that Listeria infection and vitamin and mineral intake and supplementation to be the most important. This was followed by advice regarding "general healthy nutrition", avoidance of alcohol and smoking and the importance of physical activity.

\section{b. Lack of confidence in addressing weight and GWG}

The majority of midwives thought that conversations with women regarding their weight were difficult, reflecting a negative social construction around weight. It was therefore often easier to avoid raising weight as a concern during antenatal consultations.

".weight is a difficult one. It's easier to bring it up if your blood pressure's high, or you've got protein in you urine. But when you've got to say to someone "You're a little bit overweight for midwives to look after." ..it's not a nice thing to say, but I think. . they understand if you discuss it in a clinical risk manner." (Urban midwife 6)

"I know myself I am so euphemistic about the conversation." (Urban midwife 2)

Midwives felt that it was important for them to develop the communication skills needed to establish rapport with women that would enable them to have conversations around weight so that discussions were positive, non-judgemental and did not infer blame.

"I hear young grads say all the time "oh I don't know how to talk to women cos their BMI is high" and I think to myself have you never learnt about putting your judgement to one side and giving facts and letting people see you mean what you say, that you're not there judging" (Urban midwife 7)

c. Support for midwives to promote healthy GWG

A model for education and support for midwives to increase their knowledge, skills and opportunity was the most commonly suggested way to help midwives promote and encourage healthy GWGs. Some participants 
recommended additional education and training for midwives around GWG and others saw greater opportunity for intervention which could occur during longer antenatal consultations.

"Probably for midwives to have a lot more education on what we should be saying to women and what we should be doing, because we are at the forefront of seeing these women." (Urban midwife 9)

The need for longer and individualised antenatal consultations was underpinned by the midwives' perception that women wanted individual consultations with midwives, continuity of care and relationship and trust building.

Another model to support midwives was the implementation of healthy GWG detection and management policies that would flow down to practice changes, where there was an expectation that GWG would be discussed.

"I know that's what I'd like to see, these triggers that come up. Because I know for the smoking, there's the trigger point where you must ask the questions, and it's part of what you do at every visit." (Urban midwife 6)

Models targeting women to promote healthy GWG were suggested. These models would ideally utilise multidisciplinary antenatal group sessions employing midwives, dietitians and physiotherapists. Drop in services for 'high risk' groups such as refugees and young mothers were suggested by others.

When the midwives were prompted to consider whether some of the new technologies such as the internet, telephone counselling and short message service (SMS) interventions could be used in this context, the midwives favoured the internet and SMS interventions. However, some expressed concern over quality of information and the ability for some women to access the technologies. Others felt that the introduction of these interventions may augment services, increase consistency of information and provide improved access to 'at risk' groups.

"People are hooked into the internet these days. That's where they are seeking a lot of information. "(Urban midwife 3)

“. . doesn't matter what economic class people come from, they've always got a mobile. But if it's coming to their phone, they're always going to read a message, which is a really good way to get to these people.........." (Urban midwife 9)

d. Features and content of an optimal intervention to promote healthy GWG
Continuity of care was considered an optimal feature to promote healthy GWG with women seeing the same midwives or health professionals at each visit.

"I sometimes think it's better to have that personal input from someone you've actually built up a rapport with." (Urban midwife 1)

Interventions connected and branded to the antenatal clinic and consistency of messages were seen to be central features in a contiguous approach.

\section{"..... .even hospital (nutrition based) internet sites would be good." (Urban midwife 3)}

Healthy eating, followed by physical activity and the provision of individual GWG parameters, were the topics perceived to be the most important for inclusion in an intervention. Furthermore, supporting women to learn from health providers and other women was seen to be crucial to intervention success.

". . the food group eating isn't enough, it isn't enough to tell someone who doesn't understand about nutrition that this is the way you're supposed to eat. Having someone sit down to teach them about what's on the back of packaging and how to read the packaging and what is a good food and giving them examples of what a meal is much more beneficial" (Urban midwife 10)

"Eat from a wide food group. Exercise as a balance in your life. And, I'm trying to think of the right way of putting it, don't go to extremes." (Urban midwife 7)

\section{Discussion}

In this study, midwives demonstrated a diverse range of views and practices regarding GWG, such as the detection and assessment of GWG, the provision of GWG guidelines and the understanding of the clinical significance of excess GWG. Notwithstanding, midwives universally identified some negative implications of pregnant women having excess GWG. Midwives expressed the desire to spend more time with women during antenatal clinics so they could discuss associated lifestyle behaviours. They suggested clinical interventions if excess GWG was detected and they contributed ideas regarding the ways in which they, and pregnant women, could be further supported to improve lifestyle behaviours.

The midwives' diverse, and often contradictory, views with low priority given to GWG alongside a concern for women's physical health and a belief in the role of midwives in the promotion of a healthy lifestyle, are 
corroborated by related research. In a recent UK online survey of 241 midwives, only $15 \%$ of respondents offered personalised GWG advice based on the woman's diet and physical activity [35]. This was despite 77\% believing it was appropriate and $69 \%$ believing it was feasible to offer such advice. Interestingly one challenge to providing GWG advice, suggested by this cohort, was the midwives' personal weight and weight management issues. In an older UK study differences in the perceived relevance of weighing influenced whether midwives would act in response to "abnormal" GWG, and whether they advised women to gain or lose weight during pregnancy [18]. These and other studies [20,34,39] suggest a wide variation in attitudes and practices of health providers, including midwives, regarding GWG detection, education and intervention. This may come from a lack of nationally recognised guidelines for weighing and GWG, limited health professional education and changes in practice resulting in non-evidence based approaches to care and advice.

The short and long-term implications of excess GWG on both mother and child are increasingly evident [9]. Interestingly, all the midwives interviewed acknowledged some short-term maternal complications, however, only two midwives mentioned short-term foetal health implications and one a long-term maternal complication. This finding is consistent with study of Australian GP's beliefs and practices regarding GWG [39] where GPs indicated a limited range of GWG associated complications. These findings suggest that midwives may not be fully aware of the health impacts of GWG. Further research is required to help understand the best method of increasing health providers', including midwives', understanding of the risks and complications associated with excess GWG.

This study emphasised the lifestyle behaviour priorities of the midwives. However, their priorities were at odds with the prevalence and outcome data for lifestyle behaviours during pregnancy. For example, midwives placed greater emphasis on a discussion of the prevention of Listeria contamination and the promotion of multivitamin use over general nutrition advice, physical activity and weight gain advice. These findings resonate with the UK survey of 672 midwives [18] who ranked "normal weight gain" as the least important focus of nutrition advice for pregnant women. This may reflect their understanding of, ease of delivery and comfort with various lifestyle messages. The acute versus chronic nature of sequelae is also likely to be important. A refocus of priorities is required.

Irrespective of healthy GWG, evidence suggests that women are not meeting dietary $[3,22,48]$ or physical activity [3] guidelines. Moreover, it appears that most pregnant women are seeking nutrition information [22]. More than half (55\%) of a sample of 411 Australian pregnant women, who were representative of a busy urban maternity hospital population, identified healthy eating as a priority and wanted nutrition information [22]. This illustrates the need for those working with pregnant women to reframe lifestyle behaviour education to reflect a more comprehensive view of health and wellness. Antenatal guidelines and education should be developed in the context of all lifestyle behaviours, not just Listeria prevention or GWG guidelines, to minimise the problem where particular issues are given priority over others. Research is required to develop effective interventions that address nutrition and physical activity while encompassing the specific requirements of pregnancy.

Two dominant barriers to the provision of healthy GWG and lifestyle behaviour advice identified by the midwives were a lack of confidence regarding how to provide weight advice and concern over causing psychological harm. While the concern for fostering anxiety is present in the literature $[18,20]$, the focus on this issue as a cause of active and inappropriate weight reduction remains unclear. These views may be a reflection of increasing awareness of body image and disordered eating behaviours among the population, the hospital policy from where the majority of the sample was drawn mentions that women "may try to control weight gain through inappropriate strategies" [47], and/or the desire of midwives to do "no harm" in their interactions with women. However, evidence suggests that failure to acknowledge the issue of excess weight is likely to reinforce the problem [32]. Importantly, if women are at risk of disordered eating and low GWG they would benefit from GWG guidance to prevent complications including small-for-gestational-age infants and seizure [16]. Two risk factors for gaining insufficient weight are a lack of provider advice about GWG, and provider advice to gain weight below that recommended in the guidelines [30]. Therefore, all women would benefit from weight gain advice, along with supplemented expert care for high risk groups, to make an informed choice regarding their health and GWG during pregnancy. More research is required to define the best way to approach GWG counselling for all women and ensure it is provided in a non-judgemental way and minimises stress for both parties.

Given the incomplete understandings of excessive GWG health impacts and concerns of ramifications of discussion it is therefore not surprising that only one-third of the midwives provided GWG guidelines while two-thirds did not, unless asked to do so by the woman. This, in part, may be explained by the elimination of regular weighing in antenatal practice removing a relevant cue to discuss weight. This is a concern given the evidence that suggests that the provision of guidelines appears to be successful in reducing excess GWG [27,30], meeting target GWG goals 
$[30,49]$ and thus minimising complications [5,50]. Parallels and leads to translating guidelines into practice may be found in successful antenatal smoking cessation interventions [51]. In considering the health provider's role in discussing smoking cessation, a common barrier was found to be personal beliefs that quitting smoking would have adverse effects on women's psychological wellbeing [52] and that attempting to quit resulted in inordinate expenditure of emotional energy [53]. However, many studies have demonstrated that these concerns are unfounded [54-56]. It would be timely to assess the psychological impact of GWG interventions on pregnant women.

Clear evidence based guidelines regarding weighing and GWG are urgently required to clarify the clinical utility of maternal weight measurement and to allow for consistency of practice regarding the detection and management of excess GWG. Additionally, it is important that midwives are provided with education and support regarding the implementation of GWG guidelines. Lessons again may be taken from smoking cessation intervention clinical practice guidelines. These guidelines have been specifically designed for a public maternity care setting combined with an implementation program. Their implementation resulted in an increase in evidencebased practice with some indication of improved smoking behaviour for women [57].

Future interventions promoting healthy GWG may be enhanced by viewing the issue through a new lens. Upskilling midwives regarding clinical importance and providing the tools to assess, promote and manage healthy GWG running parallel with healthy GWG interventions integrated with best-practice antenatal care were identified. Further research is required to ascertain pregnant women's attitudes and beliefs regarding GWG, healthy eating and physical activity. Such research would ensure planned interventions are tailored to meet the needs of pregnant women during a period of life whereby they are likely to be motivated to optimise the health of her unborn child. While recent meta-analyses suggest that interventions based on physical activity and dietary counselling, often combined with weight monitoring, appeared to be successful in reducing GWG [27,28], however, the evidence remains weak [23-25]. Further study is required to ascertain effective, affordable and sustainable interventions for different risk groups and settings. Internet and texting via mobile phones are two examples of such interventions.

A strength of this study was the use of in-depth interviews. Face-to-face interviews enabled participants to discuss their views in a safe and private environment, offering and validating their experiences without having to explain or justify their views to co-workers. Previous overseas studies have utilised focus groups $[27,34]$ with a mix of health providers or surveys [18,35], and these may feasibly prohibit full disclosure [42]. The regional nature of sampling allowed recruitment across many different midwifery practice areas covering a range of antenatal populations. Conversely, restricting the settings available for investigation may place some limits on the generalizability of the outcomes to wider settings. However, saturation of certain themes was obvious at an early stage in the study. The participants from different sites confirmed these themes allowing confidence in the conclusions. The regional nature of the sample must be considered when generalising to other regions and countries, however, the similarity of findings overseas $[18,20,34]$ suggests that the issues discussed by the midwives are commonplace. The length of experience of the midwives, and the recruitment of many participants from a tertiary teaching hospital, may be both a strength and a weakness of this study. The study may have benefitted from the experience of the midwives, however, it may have also skewed the results towards those with more experience.

\section{Conclusion}

This study found wide variation in midwives' views and attitudes to GWG and excess GWG. While some midwives identified excess GWG as problematic and desired more information around the issue, the majority did not view it as an important clinical problem, despite being able to identify resultant co-morbidities associated with excess GWG. All midwives felt GWG held a low level of priority in the antenatal care agenda. These are unique data in the Australian context. In addition to improving the knowledge base, these data contribute to our understanding of the opportunities and challenges in promoting healthy GWG in the public health context. Importantly this study provides a foundation for further research into the experiences of GWG for women and health providers.

\section{Additional file}

Additional file 1: Interview questions.

\section{Abbreviations}

BMI: Body mass index; GP: General practitioner; GWG: Gestational weight gain; SMS: Short message service.

\section{Competing interests}

The authors have no competing interests to report.

\section{Authors' contributions}

JW designed study, performed data collection, data analysis and drafted the manuscript. KC designed study, and contributed to data analysis and manuscript. SW contributed to data analysis and manuscript. PVP, DP and EH contributed to manuscript. All authors have critically reviewed the manuscript and approved the final manuscript. 


\section{Acknowledgements}

The authors are grateful to the midwives who willing contributed to this study.

Karen Campbell is supported by a Victorian Health Promotion Foundation Fellowship. Shelley Wilkinson is supported by an NHMRC TRIP fellowship.

\section{Author details}

${ }^{1}$ Centre for Physical Activity and Nutrition Research, School of Exercise and Nutrition Sciences, Deakin University, 221 Burwood Hwy, Burwood, Victoria 3125, Australia. ${ }^{2}$ School of Health and Social Development, Deakin University, Melbourne, Australia. ${ }^{3}$ Mercy Hospital for Women, Melbourne, Australia. ${ }^{4}$ Mater Mothers' Hospital, Brisbane, Australia. ${ }^{5}$ Mater Medical Research Institute, Brisbane, Australia.

Received: 18 May 2012 Accepted: 6 September 2012 Published: 27 September 2012

\section{References}

1. Callaway LK, Prins JB, Chang AM, Mclntyre HD: The prevalence and impact of overweight and obesity in an Australian obstetric population. Med J Aust 2006, 184(2):56-59.

2. Callaway LK, O'Callaghan MJ, McIntyre HD: Barriers to addressing overweight and obesity before conception. Med J Aust 2009, 191(8):425-428.

3. Wilkinson SA, Miller YD, Watson B: Prevalence of health behaviours in pregnancy at service entry in a Queensland health service district. Aust NZ J Publ Heal 2009, 33(3):228-233.

4. Dodd JM, Grivell RM, Nguyen A-M, Chan A, Robinson JS: Maternal and perinatal health outcomes by body mass index category. Austr NZ J Obstet Gynaecol 2011, 51(2):136-140

5. Mamun AA, Kinarivala M, O'Callaghan MJ, Williams GM, Najman JM, Callaway LK: Associations of excess weight gain during pregnancy with long-term maternal overweight and obesity: evidence from $21 \mathrm{y}$ postpartum follow-up. Am J Clin Nutr 2010, 91(5):1336-1341.

6. Kinnunen TI, Luoto R, Gissler M, Hemminki E: Pregnancy weight gain from 1960s to 2000 in Finland. Int J Obes Relat Metab Disord 2003, 27(12):1572-1577.

7. DeVader SR, Neeley HL, Myles TD, Leet TL: Evaluation of gestational weight gain guidelines for women with normal prepregnancy body mass index. Obstet Gynecol 2007, 110(4):745-751.

8. Einerson BD, Huffman JK, Istwan NB, Rhea DJ, Joy SD: New gestational weight gain guidelines: an observational study of pregnancy outcomes in obese women. Obesity 2011, 19(12):2361-2364.

9. Viswanathan M, Siega-Riz A, Moos M-K, Deierlein A, Mumford S, Knaack J, Thieda P, Lux L, Lohr K: Outcomes of maternal weight gain, Evidence Report/ Technology Assessment No.168. Rockville, MD: Prepared by RTI InternationalUniversity of North Carolina Evidence-based Practice Center under Contract No. 290-02-0016; 2008

10. Cedergren M: Effects of gestational weight gain and body mass index on obstetric outcome in Sweden. Int J Gynecol Obstet 2006, 93(3):269-274.

11. Kieffer EC, Tabaei BP, Carman WJ, Nolan GH, Guzman JR, Herman WH: The influence of maternal weight and glucose tolerance on infant birthweight in Latino mother-infant pairs. Am J Public Health 2006 96(12):2201-2208.

12. Kiel DW, Dodson EA, Artal R, Boehmer TK, Leet TL: Gestational weight gain and pregnancy outcomes in obese women: how much is enough? Obstet Gynecol 2007, 110(4):752-758.

13. Jensen DM, Ovesen P, Beck-Nielsen H, Molsted-Pedersen L, Sorensen B, Vinter C, Damm P: Gestational weight gain and pregnancy outcomes in 481 obese glucose-tolerant women. Diab Care 2005, 28(9):2118-2122.

14. Nohr EA, Vaeth M, Baker JL, Sorensen T, Olsen J, Rasmussen KM: Combined associations of prepregnancy body mass index and gestational weight gain with the outcome of pregnancy. Am J Clin Nutr 2008, 87(6):1750-1759.

15. Hilson JA, Rasmussen KM, Kjolhede CL: Excessive weight gain during pregnancy is associated with earlier termination of breast-feeding among white women. J Nutr 2006, 136(1):140-146.

16. Chen A, Feresu SA, Fernandez C, Rogan WJ: Maternal obesity and the risk of infant death in the United States. Epidemiology 2009, 20(1):74-81.

17. IOM NRC, Rasmussen K, Yaktine A: Weight gain during pregnancy: reexamining the guidelines. Washington: The National Academies; 2009

18. Ellison GT, Holliday M: The use of maternal weight measurements during antenatal care. A national survey of midwifery practice throughout the United Kingdom. J Eval Clin Pract 1997, 3(4):303-317.
19. Fraser A, Tilling K, Macdonald-Wallis C, Hughes R, Sattar N, Nelson S, Lawlor $D$ : Associations of gestational weight gain with maternal body mass index, waist circumference, and blood pressure measured 16 y after pregnancy: the Avon Longitudinal Study of Parents and Children (ALSPAC). Am J Clin Nutr 2011, 93(6):1285-1292.

20. Stotland NE, Gilbert P, Bogetz A, Harper CC, Abrams B, Gerbert B: Preventing excessive weight gain in pregnancy: how do prenatal care providers approach counseling? J Women's Health 2010, 19(4):807-814

21. Groth SW, Kearney MH: Diverse women's beliefs about weight gain in pregnancy. J Midwifery Wom Heal 2009, 54(6):452-457.

22. Wilkinson SA, Tolcher D: Nutrition and maternal health: what women want and can we provide it? Nutr Diet 2010, 67(1):18-25

23. Dodd JM, Grivell RM, Crowther CA, Robinson JS: Antenatal interventions for overweight or obese pregnant women: a systematic review of randomised trials. BJOG 2010, 117(11):1316-1326.

24. Campbell F, Messina J, Johnson M, Guillaume L, Madan J, Goyder E: Systematic review of dietary and/or physical activity interventions for weight management in pregnancy. Scheffield: The University of Scheffield; 2009.

25. Ronnberg AK, Nilsson K: Interventions during pregnancy to reduce excessive gestational weight gain: a systematic review assessing current clinical evidence using the Grading of Recommendations, Assessment, Development and Evaluation (GRADE) system. BJOG 2010 117(11):1327-1334.

26. Gardner B, Wardle J, Poston L, Croker H: Changing diet and physical activity to reduce gestational weight gain: a meta-analysis. Obes Rev 2011, 12(7):e602-e620.

27. Streuling I, Beyerlein A, von Kries R: Can gestational weight gain be modified by increasing physical activity and diet counseling? A metaanalysis of interventional trials. Am J Clin Nutr 2010, 92(4):678-687.

28. Thangaratinam S, Rogozińska $E_{1}$ Jolly K, Glinkowski S, Roseboom T, Tomlinson JW, Kunz R, Mol BW, Coomarasamy A, Khan KS: Effects of interventions in pregnancy on maternal weight and obstetric outcomes: meta-analysis of randomised evidence. BMJ 2012, 344:e2088.

29. RANZCOG: Suitability criteria for models of care and indications for referral within \& between models of care. RANZCOG 2009, C-Obs 30:1.

30. Stotland NE, Haas JS, Brawarsky P, Jackson RA, Fuentes-Afflick E, Escobar G. Body mass index, provider advice, and target gestational weight gain. Obstet Gynecol 2005, 105(3):633-638.

31. Stewart ZA, Wallace $E$, Allan C: Weight gain in pregnancy: a survey of current practices in a teaching hospital. Aus NZ J Obstet Gynaecol 2012, 52(2):208-210.

32. Heslehurst N, Lang R, Rankin J, Wilkinson JR, Summerbell CD: Obesity in pregnancy: a study of the impact of maternal obesity on NHS maternity services. BJOG 2007, 114(3):334-342

33. Heslehurst N, Moore H, Rankin J, Ells LJ, Wilkinson JR, Summberbell CD: How can maternity services be developed to effectively address maternal obesity? A qualitative study. Midwifery 2011, 27(5):e170-e177.

34. Olander EK, Atkinson L, Edmunds JK, French DP: The views of pre- and post-natal women and health professionals regarding gestational weight gain: An exploratory study. Sex Reprod Healthcare 2011, 2(1):43-48.

35. Macleod M, Gregor A, Barnett C, Magee E, Thompson J, Anderson AS: Provision of weight management advice for obese women during pregnancy: A survey of current practice and midwives' views on future approaches. Matern Child Nutr 2012, In press.

36. Sandelowski M: Whatever happened to qualitative description? Res Nurs Health 2000, 23(4):334-340.

37. Sandelowski M: What's in a name? Qualitative description revisited. Res Nurs Health 2010, 33(1):77-84.

38. Patton M: Qualitative evaluation and research methods. 2nd edition. Newbury Park, California: Sage; 1990.

39. van der Pligt P, Campbell K, Willcox J, Opie J, Denney-Wilson E: Opportunities for primary and secondary prevention of excess gestational weight gain: General Practitioners' perspectives. BMC Fam Pract 2011, 12(1):124.

40. Flick U: Triangulation in Qualitative Research. In A Companion to Qualitative Research. Edited by Flick U, von Kardoff E, Steinke I. London: Sage; 2004:178-183.

41. Fossey E, Harvey C, McDermott F, Davidson L: Understanding and evaluating qualitative research. Aust N Z J Psychiatry 2002, 36(6):717-732.

42. Liamputtong P: Qualitative data analysis: conceptual and practica considerations. Health Promot J Aust 2009, 20(2):133-139. 
43. Schmidt C: The Analysis of Semi-structured Interviews. In A Companion to Qualitative Research. Edited by Flick U, Von Kardoff E, Steinke I. London: Sage; 2004.

44. Pope C, Ziebland S, Mays N: Qualitative research in health care: analysing qualitative data. Br Med J 2000, 320(7227):114-116.

45. Thomas DR: A general inductive approach for analyzing qualitative evaluation data. Am J Eval 2006, 27(2):237-246.

46. Marques JF, McCall C: The Application of Interrater Reliability as a Solidification Instrument in a Phenomenological Study. Qual Report 2005, 10(3):439-462

47. 3 Centres Collaboration: Routine weighing at antenatal visits. Melbourne: 3 Centres Collaboration; 2010

48. Wen L, Flood VM, Simpson JM, Rissel C, Baur LA: Dietary behaviours during pregnancy: findings from first-time mothers in southwest Sydney, Australia. Int J Behav Nutr Phy 2010, 13(7):1-7.

49. Tovar A, Guthrie L, Platek D, Stuebe A, Herring S, Oken E: Modifiable predictors associated with having a gestational weight gain goal. Matern Child Hith J 2011, 15(7):1119-1126.

50. Abrams B, Altman SL, Pickett KE: Pregnancy weight gain: still controversial. Am J Clin Nutr 2000, 71(5 Suppl):1233S-1241S.

51. Lumley J, Chamberlain C, Dowswell T, Oliver S, Oakley L, Watson L: Interventions for promoting smoking cessation during pregnancy (Review). Wiley: The Cochrane Library; 2009.

52. Oliver S: Exploring lay perspectives on questions of effectiveness. In Nonrandom reflections on health services research. Edited by Maynard A, Chalmers I. London: BMJ Publishing: 1997:272-291.

53. Ebert LM, Fahy K: Why do women continue to smoke in pregnancy? Women and Birth 2007, 20(4):161-168.

54. Aveyard P, West R: Managing smoking cessation. Br Med J 2007, 335(7609):37-41.

55. Solomon LJ, Higgins ST, Heil SH, Badger GJ, Mongeon JA, Bernstein IM: Psychological symptoms following smoking cessation in pregnant smokers. J Behav Med 2006, 29(2):151-160.

56. Rigotti NA, Park ER, Regan S, Chang Y, Perry K, Loudin B, Quinn V: Efficacy of telephone counseling for pregnant smokers: a randomized controlled trial. Obstet Gynecol 2006, 108(1):83-92.

57. Flenady V, MacPhail J, New K, Devenish-Meares P, Smith J: Implementation of a clinical practice guideline for smoking cessation in a public antenatal care setting. Aust N Z J Obstet Gynaecol 2008, 48(6):552-558.

doi:10.1186/1471-2393-12-102

Cite this article as: Willcox et al:: Excess gestational weight gain: an exploration of midwives' views and practice. BMC Pregnancy and Childbirth 2012 12:102

\section{Submit your next manuscript to BioMed Central and take full advantage of:}

- Convenient online submission

- Thorough peer review

- No space constraints or color figure charges

- Immediate publication on acceptance

- Inclusion in PubMed, CAS, Scopus and Google Scholar

- Research which is freely available for redistribution 